\title{
The Effect of Stride Frequency on Running Economy and Running Distance During High Intensity Treadmill Running
}

\author{
Boram Lim \& Young Sub Kwon² \\ 1 University of Nevada, Las Vegas, USA \\ 2 Humboldt State University, California, USA
}

\begin{abstract}
Running economy (RE; $\mathrm{ml}^{\mathrm{kg}} \mathrm{g}^{-1} \mathrm{~km}^{\prime}$ ) considers as a valid predictor of endurance running performance. Theoretically, improving RE allows runners to cover more distance at constant speed or run faster at a given distance. Stride frequency (SF) is one of the important parameters that affects running performance. The purpose of this study was to investigate the effect of SF on RE and distance while running on the treadmill at the speed of $\mathrm{VO}_{2} \max \left(\mathrm{SVO}_{2} \mathrm{max}\right)$ until volitional fatigue. The second purpose was to determine a relationship between RE and running distance during high intensity running. We hypothesized that both RE and running distance would affect significantly by SF variations and there is a positive relationship between RE and running distance.
\end{abstract}

Keywords: running economy, stride frequency, high intensity running performance

$\begin{array}{lr}\text { Article History } & \text { Corresponding Author } \\ \text { Received } 20 \text { September } 2020 & \text { Boram Lim } \\ \text { Accepted } 20 \text { October } 2020 & \text { boram.lim@unlv.edu } \\ \text { Published } 31 \text { January 2021 } & \text { Department of Kinesiology and Nutrition Sciences } \\ \text { Available online 19 February 2021 } & \text { School of Integrated Health Sciences } \\ \text { https://doi.org/10.47544/johsk.2021.2.1.13 } & \text { University of Nevada, Las Vegas, USA }\end{array}$

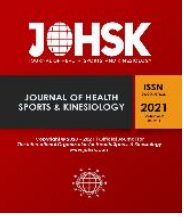

\section{Methods}

Ten male recreational runners (age: $25.8 \pm 5.0 \mathrm{yrs}$, height: $171.4 \pm 6.2 \mathrm{~cm}$, mass: $71.9 \pm 7.5 \mathrm{~kg}$ ) completed total seven experimental sessions including graded exercise test and running session for determining $\mathrm{VO}_{2} \mathrm{max}(55.4 \pm 5.9 \mathrm{ml} \mathrm{kg}$ 1 min') and preferred SF (PSF; 88.0 \pm 3.9 strides/min), respectively. Running speed was calculated based on $\mathrm{VO}_{2} \mathrm{max}$ using the metabolic equation; $\mathrm{VO}_{2}\left(\mathrm{ml}^{\mathrm{kg}} \mathrm{kg}^{-1} \cdot \mathrm{min}^{1}\right)=[0.2 \times \mathrm{Speed}(\mathrm{m} / \mathrm{min})]+3.5\left(\mathrm{ml}^{\mathrm{kg}} \mathrm{kg}^{-1} \cdot \mathrm{min}^{1}\right)$. Participants performed five separate running sessions (PSF, $\pm 5 \%, \pm 10 \%$ ) on the treadmill at the $s \mathrm{O}_{2}$ max with $0 \%$ gradient until volitional fatigue. A computer-based metronome was played in order to help maintain a target SF while running.

\section{Results}

The running distance was significantly different among SF variations $(p<0.01)$ and all participants ran the greater distance at $105 \%$ PSF. However, RE was not statistically significant $(p=0.19)$ across the SF conditions. There was a low correlation between RE and running distance $(r=0.214, p=0.14)$.

\section{Journal of Health, Sports, \& Kinesiology | ISSN 2692-9864 | www.johsk.com}


| 2021 | Volume 2 | Issue 1 | The Journal of Health, Sports, and Kinesiology |

\section{Discussion}

SF variations have a significant influence on running distance, but not RE during high intensity running. Recretional runners may use $105 \%$ PSF during high intensity running to train both aerobic and anaerobic capacity.

\section{Journal of Health, Sports, \& Kinesiology | ISSN 2692-9864 | www.johsk.com}

